\title{
Research Article \\ On Extreme Residual Lives after the Failure of the System
}

\author{
Serkan Eryilmaz ${ }^{1}$ and Ismihan Bayramoglu ${ }^{2}$ \\ ${ }^{1}$ Department of Industrial Engineering, Atilim University, Incek, 06836 Ankara, Turkey \\ ${ }^{2}$ Department of Mathematics, Izmir University of Economics, 35330 Izmir, Turkey
}

Correspondence should be addressed to Serkan Eryilmaz, seryilmaz@atilim.edu.tr

Received 25 January 2012; Revised 18 May 2012; Accepted 20 May 2012

Academic Editor: Alexander Pogromsky

Copyright (c) 2012 S. Eryilmaz and I. Bayramoglu. This is an open access article distributed under the Creative Commons Attribution License, which permits unrestricted use, distribution, and reproduction in any medium, provided the original work is properly cited.

The concept of residual lifetime has attracted considerable research interest in reliability theory. It is useful for evaluating the dynamic behavior of a system. In this paper, we study the extreme residual lives, that is, the minimum and maximum residual lives of the remaining components after the failure of the system. The system is assumed to have an arbitrary structure. We obtain signature-based distributional and ordering results for the extreme residual lives.

\section{Introduction}

A considerable attention has been given to the concept of residual lifetime in reliability and survival analysis. There are various works in the literature not only on the residual lifetime of systems but also on their components. See, for example Asadi and Bayramoglu [1], Navarro et al. [2], Bairamov and Arnold [3], Asadi and Goliforushani [4], Sadegh [5], and Eryilmaz [6].

In this paper, we study the extreme residual lifetimes of the remaining components after the complete failure of the system. These extremes might be useful to determine if the remaining components should be used after repair in the renewed system. For example, a decision maker may suggest the reuse of the remaining components if the minimum residual live is above a given threshold, or the refuse of them if the maximum residual live is below a given threshold. We aim to study the marginal and joint distributions of extreme residual lives for an arbitrary coherent structure. Our results are based on system signature, so we can easily evaluate these random variables for a given structure with a known signature.

Consider a coherent system with the lifetime $T=\phi\left(X_{1}, \ldots, X_{n}\right)$, where $X_{1}, \ldots, X_{n}$ are independent and identically distributed (iid) random variables representing the lifetimes of 
components, and have common absolutely continuous distribution $F$ and density $f$. It is well known that the survival function of $T$ can be written as

$$
P\{T>t\}=\sum_{i=1}^{n} p_{i} P\left\{X_{i: n}>t\right\}
$$

where $X_{i: n}$ is the $i$ th smallest lifetime among $X_{1}, \ldots, X_{n}$ and $p_{i}=P\left\{T=X_{i: n}\right\}, i=1, \ldots, n$. In words, $p_{i}$ is the proportion of permutations among the $n$ ! equally likely permutations of $X_{1}, \ldots, X_{n}$ that result in a minimal cut set failure when $i$ components break down. More explicitly,

$$
p_{i}=\frac{\# \text { of orderings for which the } i \text { th failure causes system failure }}{n !},
$$

$i=1, \ldots, n$. The vector $\mathbf{p}=\left(p_{1}, \ldots, p_{n}\right)$ defines a discrete probability distribution and is called system signature. The computation of $\mathbf{p}$ is a well-defined combinatorial problem. The $i$ th element of $\mathbf{p}$ can be computed from

$$
p_{i}=a_{n-i+1}(n)-a_{n-i}(n),
$$

where

$$
a_{i}(n)=\frac{r_{i}(n)}{\left(\begin{array}{c}
n \\
i
\end{array}\right)}
$$

$i=1, \ldots, n$, and $r_{i}(n)$ is the number of path sets of the structure including $i$ working components (see, e.g., Boland [7]).

The concept of system signature is a useful tool for the evaluation of reliability characteristics and ordering properties of coherent systems. An extensive review of the signature of coherent system and its applications can be found in Samaniego [8].

Let $N$ be a random variable which represents the number of surviving components at the time when the system with lifetime $T$ fails. It is known that

$$
P\{N=n-i\}=P\left\{T=X_{i: n}\right\}=p_{i}
$$

for $i=1, \ldots, n-1$ (Eryilmaz [9]). Let $X_{i}^{\prime}$ denote the residual lifetime of a surviving component after the failure of the system, that is

$$
X_{i}^{\prime} \stackrel{\text { st }}{=}\left(X_{i}-T \mid X_{i}>T\right),
$$

for $i=1, \ldots, N$.

Bairamov and Arnold [3] studied the joint distribution of the residual lifetimes of the remaining (surviving) components after the $k$ th failure in the system, that is when the system has $k$-out-of- $n$ :F structure. In this case $P\left\{T=X_{k: n}\right\}=1$, and hence the remaining number of 
components is $n-k$, that is, $P\{N=n-k\}=1$. They have shown that the joint survival function of the residual life lengths is

$$
P\left\{X_{1}^{\prime}>x_{1}, \ldots, X_{n-k}^{\prime}>x_{n-k}\right\}=\int_{0}^{\infty}\left[\prod_{j=1}^{n-k} \frac{\bar{F}\left(x_{j}+t\right)}{\bar{F}(t)}\right] d F_{k: n}(t),
$$

where

$$
d F_{k: n}(t)=\frac{n !}{(k-1) !(n-k) !} F^{k-1}(t)(1-F(t))^{n-k} d F(t)
$$

In the present paper, we study the residual lifetimes of the remaining components, in particular, minimum and maximum residual lives for an arbitrary coherent structure. The paper is organized as follows. In Section 2, we obtain mixture representations for the marginal and joint distributions of minimum and maximum residual lives. Section 3 contains stochastic ordering results on the minimum and maximum residual lives of two systems having different structures. Finally in Section 4, we present illustrative examples.

\section{Extreme Residual Lives}

Obviously, for an arbitrary coherent structure (different from $k$-out-of- $n$ :F structure), the number of surviving components is a random variable. Thus, in general, it should be taken into account that we have random number of surviving components on hand after the failure of the system. This case is illustrated in the following example.

Example 2.1. Consider the system which functions if and only if at least two consecutive of $n=6$ components function. That is, the system has consecutive 2-out-of-6:G structure. The lifetime of this system is given by the following:

$$
T=\max \left(\min \left(X_{1}, X_{2}\right), \min \left(X_{2}, X_{3}\right), \min \left(X_{3}, X_{4}\right), \min \left(X_{4}, X_{5}\right), \min \left(X_{5}, X_{6}\right)\right) .
$$

For a consecutive $k$-out-of- $n: G$ system, it is known that

$$
r_{i}(n)=\left(\begin{array}{c}
n \\
i
\end{array}\right)-\sum_{j=0}^{\min ([i / k], n-i+1)}(-1)^{j}\left(\begin{array}{c}
n-i+1 \\
j
\end{array}\right)\left(\begin{array}{c}
n-j k \\
n-i
\end{array}\right)
$$

where $[x]$ denotes the integer part of $x$ (see, e.g., Salehi et al. [10]). Using (2.2) for $n=6$ and $k=2$ in (1.3), the signature of consecutive 2-out-of- $6: \mathrm{G}$ system is found to be the following:

$$
p=\left(0,0, \frac{3}{15}, \frac{7}{15}, \frac{5}{15}, 0\right)
$$


Table 1: $E(N)$ for consecutive 2-out-of- $n: G$ systems.

\begin{tabular}{lr}
\hline$n$ & $E(N)$ \\
\hline 20 & 3.5737 \\
30 & 4.4445 \\
50 & 5.8368 \\
\hline
\end{tabular}

Therefore, at the end of the lifetime of the system we may have 1, 2, or 3 surviving components with respective probabilities as follows:

$$
P\{N=1\}=\frac{5}{15}, \quad P\{N=2\}=\frac{7}{15}, \quad P\{N=3\}=\frac{3}{15},
$$

and hence the expected number of surviving components at the time when the system fails is $E(N) \cong 2$.

In Table 1, we compute $E(N)$ for consecutive 2-out-of- $n: G$ system whose lifetime is defined by the following:

$$
T=\max _{1 \leq i \leq n-1} \min \left(X_{i}, X_{i+1}\right) .
$$

In view of Table 1 , we observe that $E(N)$ depends on $n$, and there is no limiting point for $E(N)$ when $n$ tends to infinity.

The minimum and maximum residual lifetimes after the failure of the system are defined respectively as follows:

$$
\begin{aligned}
X_{1: N}^{\prime} & =\min \left(X_{1}^{\prime}, \ldots, X_{N}^{\prime}\right), \\
X_{N: N}^{\prime} & =\max \left(X_{1}^{\prime}, \ldots, X_{N}^{\prime}\right),
\end{aligned}
$$

where $X_{1}^{\prime}, \ldots, X_{N}^{\prime}$ represent the residual lifetimes of the remaining components. Obviously, the problem is ridiculous for a system satisfying $P\{N=0\}=P\left\{T=X_{n: n}\right\}=p_{n}>0$. Thus we consider the systems satisfying $P\{N>0\}=1$ or equivalently $p_{n}=0$.

In the following Theorem, we obtain mixture representations for the distributions of the random variables $X_{1: N}^{\prime}$ and $X_{N: N}^{\prime}$.

Theorem 2.2. Let $T=\phi\left(X_{1}, \ldots, X_{n}\right)$ denote the lifetime of a coherent system with iid components and signature $\mathbf{p}=\left(p_{1}, \ldots, p_{n}\right)$ satisfying $p_{n}=0$. Then

$$
\begin{gathered}
P\left\{X_{1: N}^{\prime} \leq x\right\}=\sum_{i=1}^{n-1} p_{i} P\left\{X_{1: n-i}^{\prime} \leq x\right\}, \\
P\left\{X_{N: N}^{\prime} \leq x\right\}=\sum_{i=1}^{n-1} p_{i} P\left\{X_{n-i: n-i}^{\prime} \leq x\right\}
\end{gathered}
$$

where $X_{1: n-i}^{\prime}$ and $X_{n-i: n-i}^{\prime}$ are the minimum and maximum order statistics corresponding to exchangeable random variables $X_{1}^{\prime}, \ldots, X_{n-i}^{\prime}$. 
Proof. For a coherent system with lifetime $T=\phi\left(X_{1}, \ldots, X_{n}\right)$, via the order statistic equivalent of $T$, one obtains

$$
\begin{aligned}
P\left\{X_{1}^{\prime}\right. & \left.>x_{1}, \ldots, X_{N}^{\prime}>x_{N}\right\} \\
& =\sum_{i=1}^{n-1} P\left\{X_{1}^{\prime}>x_{1}, \ldots, X_{n-i}^{\prime}>x_{n-i} \mid T=X_{i: n}\right\} P\left\{T=X_{i: n}\right\} .
\end{aligned}
$$

The conditional probability in the last equation is actually the probability given in (1.7) by replacing $k$ by $i$. Thus we have

$$
P\left\{X_{1}^{\prime}>x_{1}, \ldots, X_{N}^{\prime}>x_{N}\right\}=\sum_{i=1}^{n-1} p_{i} \int_{0}^{\infty}\left[\prod_{j=1}^{n-i} \frac{\bar{F}\left(x_{j}+t\right)}{\bar{F}(t)}\right] d F_{i: n}(t) .
$$

Similarly, we can obtain

$$
P\left\{X_{1}^{\prime} \leq x_{1}, \ldots, X_{N}^{\prime} \leq x_{N}\right\}=\sum_{i=1}^{n-1} p_{i} \int_{0}^{\infty}\left[\prod_{j=1}^{n-i} \frac{F\left(x_{j}+t\right)-F(t)}{\bar{F}(t)}\right] d F_{i: n}(t) .
$$

The results of the theorem follow immediately because

$$
\begin{aligned}
P\left\{X_{1: N}^{\prime} \leq x\right\} & =1-P\left\{X_{1}^{\prime}>x, \ldots, X_{N}^{\prime}>x\right\} \\
& =1-\sum_{i=1}^{n-1} p_{i} \int_{0}^{\infty}\left[\frac{\bar{F}(x+t)}{\bar{F}(t)}\right]^{n-i} d F_{i: n}(t) \\
& =1-\sum_{i=1}^{n-1} p_{i} P\left\{X_{1: n-i}^{\prime}>x\right\} \\
P\left\{X_{N: N}^{\prime} \leq x\right\} & =P\left\{X_{1}^{\prime} \leq x, \ldots, X_{N}^{\prime} \leq x\right\} \\
& =\sum_{i=1}^{n-1} p_{i} \int_{0}^{\infty}\left[\frac{F(x+t)-F(t)}{\bar{F}(t)}\right]^{n-i} d F_{i: n}(t) \\
& =\sum_{i=1}^{n-1} p_{i} P\left\{X_{n-i: n-i}^{\prime} \leq x\right\} .
\end{aligned}
$$

Under the assumption that the component lifetime distribution is exponential, the distributions of the random variables $X_{1: N}^{\prime}$ and $X_{N: N}^{\prime}$ can be written as mixtures of the distributions of order statistics corresponding to $X_{1}, \ldots, X_{n}$. This is due to the independence of residual lifetimes and the preservation of the original lifetime distribution of a component in the case of exponential distribution. That is, the residual lifetime distribution of a component is same as the original lifetime distribution of a component. The results are presented in the following Corollary. 
Corollary 2.3. Let $X_{1}, \ldots, X_{n}$ be iid lifetime random variables with common $c d f F(t)=1-$ $\exp (-\lambda t), t \geq 0$. Then

$$
\begin{gathered}
P\left\{X_{1: N}^{\prime} \leq x\right\}=\sum_{i=1}^{n-1} p_{i} P\left\{X_{1: n-i} \leq x\right\}, \\
P\left\{X_{N: N}^{\prime} \leq x\right\}=\sum_{i=1}^{n-1} p_{i} P\left\{X_{n-i: n-i} \leq x\right\} .
\end{gathered}
$$
$X_{N: N}^{\prime}$

In the following, we present the joint distribution of the random variables $X_{1: N}^{\prime}$ and

Theorem 2.4. Let $T=\phi\left(X_{1}, \ldots, X_{n}\right)$ denote the lifetime of a coherent system with iid components and signature $\mathbf{p}=\left(p_{1}, \ldots, p_{n}\right)$ satisfying $p_{n}=0$. Then

$$
P\left\{X_{1: N}^{\prime} \leq x, X_{N: N}^{\prime} \leq y\right\}=\sum_{i=1}^{n-1} p_{i} P\left\{X_{1: n-i}^{\prime} \leq x, X_{n-i: n-i}^{\prime} \leq y\right\}
$$

for $x<y$.

Proof. For $x<y$, it is obvious that

$$
P\left\{X_{1: N}^{\prime} \leq x, X_{N: N}^{\prime} \leq y\right\}=P\left\{X_{N: N}^{\prime} \leq y\right\}-P\left\{X_{1: N}^{\prime}>x, X_{N: N}^{\prime} \leq y\right\}
$$

By conditioning on $N$,

$$
\begin{aligned}
P\left\{X_{1: N}^{\prime}\right. & \left.>x, X_{N: N}^{\prime} \leq y\right\} \\
& =\sum_{i=1}^{n-1} P\left\{x<X_{1}^{\prime} \leq y, \ldots, x<X_{n-i}^{\prime} \leq y \mid T=X_{i: n}\right\} P\left\{T=X_{i: n}\right\} .
\end{aligned}
$$

It is easy to see that

$$
\begin{aligned}
P\{x & \left.<X_{1}^{\prime} \leq y, \ldots, x<X_{n-i}^{\prime} \leq y \mid T=X_{i: n}\right\} \\
& =\int_{0}^{\infty}\left[\frac{F(y+t)-F(x+t)}{\bar{F}(t)}\right]^{n-i} d F_{i: n}(t) \\
& =P\left\{X_{1: n-i}^{\prime}>x, X_{n-i: n-i}^{\prime} \leq y\right\} .
\end{aligned}
$$

Therefore,

$$
\begin{aligned}
P\left\{X_{1: N}^{\prime}\right. & \left.\leq x, X_{N: N}^{\prime} \leq y\right\} \\
& =\sum_{i=1}^{n-1} p_{i} P\left\{X_{n-i: n-i}^{\prime} \leq y\right\}-\sum_{i=1}^{n-1} p_{i} P\left\{X_{1: n-i}^{\prime}>x, X_{n-i: n-i}^{\prime} \leq y\right\},
\end{aligned}
$$

which completes the proof. 
Corollary 2.5. Let $X_{1}, \ldots, X_{n}$ be iid lifetime random variables with common $c d f F(t)=1$ $\exp (-\lambda t), t \geq 0$. Then

$$
P\left\{X_{1: N}^{\prime} \leq x, X_{N: N}^{\prime} \leq y\right\}=\sum_{i=1}^{n-1} p_{i} P\left\{X_{1: n-i} \leq x, X_{n-i: n-i} \leq y\right\}
$$

for $x<y$.

\section{Stochastic Ordering Results}

Let us consider two systems with different structures having lifetimes $T_{1}=\phi_{1}\left(X_{1}, \ldots, X_{n}\right)$, and $T_{2}=\phi_{2}\left(X_{1}, \ldots, X_{n}\right)$. The respective signatures of the systems are defined by the following:

$$
\begin{aligned}
& p_{i}=P\left\{T_{1}=X_{i: n}\right\}=P\left\{N_{1}=n-i\right\}, \\
& q_{i}=P\left\{T_{2}=X_{i: n}\right\}=P\left\{N_{2}=n-i\right\},
\end{aligned}
$$

for $i=1, \ldots, n-1$.

The residual lifetimes of the remaining components in systems $\phi_{1}$ and $\phi_{2}$ are defined respectively, as follows

$$
\begin{array}{ll}
X_{i}^{\prime}=\left(X_{i}-T_{1} \mid X_{i}>T_{1}\right), & i=1, \ldots, N_{1} \\
Y_{i}^{\prime}=\left(X_{i}-T_{2} \mid X_{i}>T_{2}\right), & i=1, \ldots, N_{2} .
\end{array}
$$

Although $X_{i}$ s and $Y_{i}$ s have different joint distributions, given $\left\{T_{1}=X_{i: n}\right\}$ and $\left\{T_{2}=X_{i: n}\right\}$ they have the same joint distributions. If $X_{1: N_{1}}^{\prime}\left(Y_{1: N_{2}}^{\prime}\right)$ and $X_{N_{1}: N_{1}}^{\prime}\left(Y_{N_{2}: N_{2}}^{\prime}\right)$ denote, respectively, the minimum and maximum residual lives corresponding to the system $\phi_{1}\left(\phi_{2}\right)$, then using Theorem 2.2 we have

$$
\begin{aligned}
& P\left\{X_{1: N_{1}}^{\prime} \leq x\right\}=\sum_{i=1}^{n-1} p_{i} P\left\{X_{1: n-i}^{\prime} \leq x\right\}, \\
& P\left\{Y_{1: N_{2}}^{\prime} \leq x\right\}=\sum_{i=1}^{n-1} q_{i} P\left\{Y_{1: n-i}^{\prime} \leq x\right\},
\end{aligned}
$$

where $X_{1: n-i}^{\prime}$ and $Y_{1: n-i}^{\prime}$ are the minimum residual lives after the failure of the $i$ th component, that is after time $X_{i: n}$. Since the systems have the common components $X_{1: n-i}^{\prime} \stackrel{d}{=} Y_{1: n-i}^{\prime}$. Thus $P\left\{Y_{1: N_{2}}^{\prime} \leq x\right\}$ can be equivalently written as follows

$$
P\left\{Y_{1: N_{2}}^{\prime} \leq x\right\}=\sum_{i=1}^{n-1} q_{i} P\left\{X_{1: n-i}^{\prime} \leq x\right\}
$$


That is, the distributions of $X_{1: N_{1}}^{\prime}$ and $Y_{1: N_{2}}^{\prime}$ differ from each other through systems' structures and this is taken into account by the coefficients of $P\left\{X_{1: n-i}^{\prime} \leq x\right\}$.

For two discrete distributions $\mathbf{p}=\left(p_{1}, \ldots, p_{n}\right)$ and $\mathbf{q}=\left(q_{1}, \ldots, q_{n}\right)$, let $\leq_{\mathrm{st}}, \leq_{\mathrm{hr}}$, and $\leq_{\mathrm{rh}}$ represent, respectively, the usual stochastic order, hazard rate order, and reversed hazard rate order. Then

(a) $\mathbf{p} \leq_{\mathrm{st}} \mathbf{q}$ if $\sum_{j=i}^{n} p_{j} \leq \sum_{j=i}^{n} q_{j}$ for all $i=1,2, \ldots, n$.

(b) $\mathbf{p} \leq_{\mathrm{hr}} \mathbf{q}$ if $\sum_{j=i}^{n} p_{j} / \sum_{j=i}^{n} q_{j}$ is decreasing in $i$.

(c) $\mathbf{p} \leq_{\mathrm{rh}} \mathbf{q}$ if $\sum_{j=1}^{i} p_{j} / \sum_{j=1}^{i} q_{j}$ is decreasing in $i$.

Theorem 3.1. If $\mathbf{p} \leq_{\mathrm{st}} \mathbf{q}$, then $X_{1: N_{1}}^{\prime} \leq_{\mathrm{st}} Y_{1: N_{2}}^{\prime}$ and $X_{N_{1}: N_{1}}^{\prime} \geq_{\mathrm{st}} Y_{N_{2}: N_{2}}^{\prime}$.

Proof. Because $X_{1: n-i}^{\prime} \leq_{\mathrm{st}} X_{1: n-i-1}^{\prime}$, the function $P\left\{X_{1: n-i}^{\prime}>x\right\}$ is nondecreasing in $i$ for all $x$. Thus if $\mathbf{p} \leq_{\text {st }} \mathbf{q}$, then

$$
\begin{aligned}
P\left\{X_{1: N_{1}}^{\prime}>x\right\} & =\sum_{i=1}^{n-1} p_{i} P\left\{X_{1: n-i}^{\prime}>x\right\} \\
& \leq \sum_{i=1}^{n-1} q_{i} P\left\{X_{1: n-i}^{\prime}>x\right\}=P\left\{Y_{1: N_{2}}^{\prime}>x\right\}
\end{aligned}
$$

which implies $X_{1: N_{1}}^{\prime} \leq_{\text {st }} Y_{1: N_{2}}^{\prime}$. On the other hand, $X_{n-i: n-i}^{\prime} \geq_{\text {st }} X_{n-i-1: n-i-1}^{\prime}$ and hence $P\left\{X_{n-i: n-i}^{\prime} \leq\right.$ $x$ \} is nondecreasing in $i$ for all $x$. Thus if $\mathbf{p} \leq_{\text {st }} \mathbf{q}$, then

$$
\begin{aligned}
P\left\{X_{N_{1}: N_{1}}^{\prime} \leq x\right\} & =\sum_{i=1}^{n-1} p_{i} P\left\{X_{n-i: n-i}^{\prime} \leq x\right\} \\
& \leq \sum_{i=1}^{n-1} q_{i} P\left\{X_{n-i: n-i}^{\prime} \leq x\right\}=P\left\{Y_{N_{2}: N_{2}}^{\prime} \leq x\right\},
\end{aligned}
$$

which implies $X_{N_{1}: N_{1}}^{\prime} \geq_{\text {st }} Y_{N_{2}: N_{2}}^{\prime}$.

Lemma 3.2 (see [11]). Let $\alpha$ and $\beta$ be two real valued functions such that $\beta$ is nonnegative and $\alpha / \beta$ and $\beta$ are nondecreasing. If $X_{i}$ has distribution $F_{i}, i=1,2$ and $X_{1} \leq{ }_{h r} X_{2}$, then

$$
\frac{\int_{-\infty}^{\infty} \alpha(x) d F_{1}(x)}{\int_{-\infty}^{\infty} \beta(x) d F_{1}(x)} \leq \frac{\int_{-\infty}^{\infty} \alpha(x) d F_{2}(x)}{\int_{-\infty}^{\infty} \beta(x) d F_{2}(x)} .
$$

Lemma 3.3 (see [12]). Let $\alpha$ and $\beta$ be two real valued functions such that $\alpha$ is nonnegative and $\beta / \alpha$ and $\alpha$ are nonincreasing. If $X_{i}$ has distribution $F_{i}, i=1,2$ and $X_{1} \leq_{\mathrm{rh}} X_{2}$, then

$$
\frac{\int_{-\infty}^{\infty} \beta(x) d F_{1}(x)}{\int_{-\infty}^{\infty} \alpha(x) d F_{1}(x)} \geq \frac{\int_{-\infty}^{\infty} \beta(x) d F_{2}(x)}{\int_{-\infty}^{\infty} \alpha(x) d F_{2}(x)} .
$$


Theorem 3.4. (a) If $\mathbf{p} \leq \mathrm{hr} \mathbf{q}$, and $X_{1: n-i}^{\prime} \leq{ }_{\mathrm{hr}} X_{1: n-i-1}^{\prime}$ for $i=1, \ldots, n-2$, then $X_{1: N_{1}}^{\prime} \leq_{\mathrm{hr}} Y_{1: N_{2}}^{\prime}$.

(b) If $\mathbf{p} \leq_{\mathrm{rh}} \mathbf{q}$, and $X_{n-i: n-i}^{\prime} \geq{ }_{\mathrm{hr}} X_{n-i-1: n-i-1}^{\prime}$ for $i=1, \ldots, n-2$, then $X_{N_{1}: N_{1}}^{\prime} \geq_{h r} Y_{N_{2}: N_{2}}^{\prime}$.

Proof. Because $X_{1: n-i}^{\prime} \leq h r X_{1: n-i-1}^{\prime}, P\left\{X_{1: n-i-1}^{\prime}>x\right\} / P\left\{X_{1: n-i}^{\prime}>x\right\}$ is nondecreasing in $x$. That is, for $x_{1} \leq x_{2}$

$$
\frac{P\left\{X_{1: n-i-1}^{\prime}>x_{2}\right\}}{P\left\{X_{1: n-i-1}^{\prime}>x_{1}\right\}} \geq \frac{P\left\{X_{1: n-i}^{\prime}>x_{2}\right\}}{P\left\{X_{1: n-i}^{\prime}>x_{1}\right\}} .
$$

which implies that $P\left\{X_{1: n-i}^{\prime}>x_{2}\right\} / P\left\{X_{1: n-i}^{\prime}>x_{1}\right\}$ is nondecreasing in $i$. Applying Lemma 3.2, and using $\mathbf{p} \leq \mathrm{hr} \mathbf{q}$ we obtain

$$
\frac{\sum_{i=1}^{n-1} q_{i} P\left\{X_{1: n-i}^{\prime}>x_{2}\right\}}{\sum_{i=1}^{n-1} p_{i} P\left\{X_{1: n-i}^{\prime}>x_{2}\right\}} \geq \frac{\sum_{i=1}^{n-1} q_{i} P\left\{X_{1: n-i}^{\prime}>x_{1}\right\}}{\sum_{i=1}^{n-1} p_{i} P\left\{X_{1: n-i}^{\prime}>x_{1}\right\}} .
$$

for $x_{1} \leq x_{2}$, which implies that $X_{1: N_{1}}^{\prime} \leq \mathrm{hr} Y_{1: N_{2}}^{\prime}$. The proof of part (b) is based on similar arguments and the usage of Lemma 3.3.

As stated before, if the original lifetime distribution is exponential, then the residual lifetimes are iid. For a sequence of iid random variables $X_{1}, \ldots, X_{n}$ we have $X_{1: m+1} \leq_{h r} X_{1: m}$, and $X_{m: m} \leq \mathrm{hr} X_{m+1: m+1}, m=1,2, \ldots$. Thus, the problem of comparing extreme residual lives reduces to the comparison of systems' signatures for the exponential lifetime distribution and we obtain the following Corollary.

Corollary 3.5. Let $X_{1}, \ldots, X_{n}$ be iid lifetime random variables with common $c d f F(t)=1-$ $\exp (-\lambda t), t \geq 0$. Then

(a) If $\mathbf{p} \leq \mathrm{hr} \mathbf{q}$, then $X_{1: N_{1}}^{\prime} \leq{ }_{\mathrm{hr}} Y_{1: N_{2}}^{\prime}$.

(b) If $\mathbf{p} \leq_{\mathrm{rh}} \mathbf{q}$, then $X_{N_{1}: N_{1}}^{\prime} \geq_{\mathrm{hr}} Y_{N_{2}: N_{2}}^{\prime}$.

\section{Illustrative Examples}

Consider the systems with structure functions

$$
\begin{gathered}
\phi_{1}\left(x_{1}, x_{2}, x_{3}, x_{4}, x_{5}\right)=\max \left(\min \left(x_{1}, x_{2}, x_{3}\right), \min \left(x_{1}, x_{4}, x_{5}\right)\right), \\
\phi_{2}\left(x_{1}, x_{2}, x_{3}, x_{4}, x_{5}\right)=\max \left(\min \left(x_{1}, x_{2}, x_{3}\right), \min \left(x_{1}, x_{2}, x_{4}\right), \min \left(x_{1}, x_{3}, x_{5}\right)\right) .
\end{gathered}
$$

The respective signatures of these systems are $\mathbf{p}=(1 / 5,3 / 5,1 / 5,0,0)$, and $\mathbf{q}=$ $(1 / 5,1 / 2,3 / 10,0,0)$ (see Navarro and Rubio [13]). Thus we have

$$
\begin{aligned}
& P\left\{N_{1}=2\right\}=\frac{1}{5}, \quad P\left\{N_{1}=3\right\}=\frac{3}{5}, \quad P\left\{N_{1}=4\right\}=\frac{1}{5}, \\
& P\left\{N_{2}=2\right\}=\frac{3}{10}, \quad P\left\{N_{2}=3\right\}=\frac{1}{2}, \quad P\left\{N_{2}=4\right\}=\frac{1}{5} .
\end{aligned}
$$




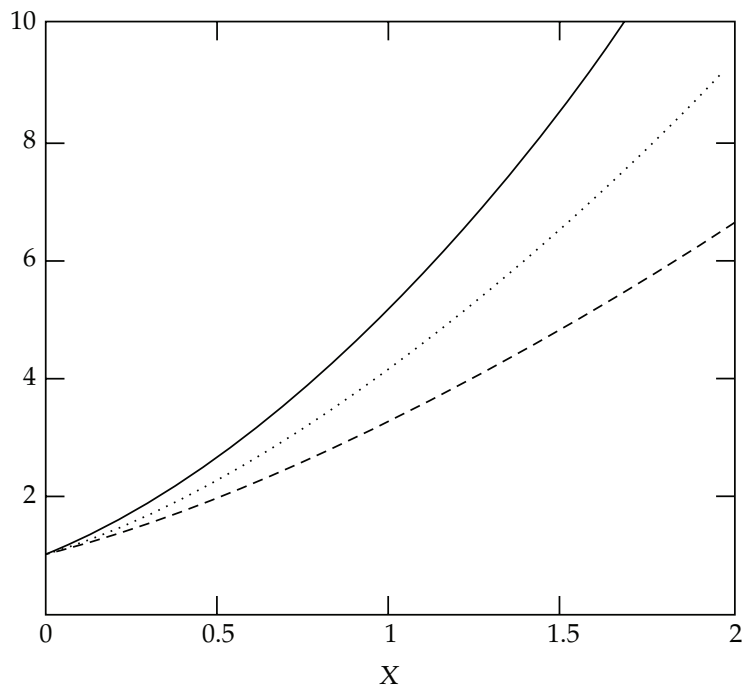

Figure 1: Plot of the ratio $r_{n}(i, x)$ for $i=1,2,3$ from the above to below.

Table 2: Expected values of the extreme residual lives.

\begin{tabular}{ccccc}
\hline$\alpha$ & $E\left(X_{1: N_{1}}^{\prime}\right)$ & $E\left(Y_{1: N_{2}}^{\prime}\right)$ & $E\left(X_{N_{1}: N_{1}}^{\prime}\right)$ & $E\left(Y_{N_{2}: N_{2}}^{\prime}\right)$ \\
\hline 2 & 0.2857 & 0.3111 & 2.7746 & 2.7492 \\
3 & 0.1597 & 0.1714 & 1.1883 & 1.1737 \\
4 & 0.1103 & 0.1176 & 0.7373 & 0.7271 \\
5 & 0.0841 & 0.0893 & 0.5305 & 0.5227 \\
\hline
\end{tabular}

Because $\mathbf{p} \leq_{\mathrm{st}} \mathbf{q}$, from Theorem 3.1, we have $X_{1: N_{1}}^{\prime} \leq_{\mathrm{st}} Y_{1: N_{2}}^{\prime}$ and $X_{N_{1}: N_{1}}^{\prime} \geq_{\mathrm{st}} Y_{N_{2}: N_{2}}^{\prime}$. Suppose that the common lifetime distribution of components is Lomax with cdf $F(t)=1-(1+t)^{-\alpha}, t>$ $0, \alpha>0$. Note that for $\alpha \leq 1$, the expected value of this distribution is infinite and hence we consider the case $\alpha>1$. In Table 2, we compute the expected values of the extreme residual lives for the two systems for different values of the parameter $\alpha$.

For $\alpha=2$, from the Figure 1, we observe that

$$
r_{n}(i, x)=\frac{P\left\{X_{1: n-i-1}^{\prime}>x\right\}}{P\left\{X_{1: n-i}^{\prime}>x\right\}}
$$

is nondecreasing in $x$ for $i=1,2,3$. That is, $X_{1: 4}^{\prime} \leq \leq_{\mathrm{hr}} X_{1: 3}^{\prime}, X_{1: 3}^{\prime} \leq{ }_{\mathrm{hr}} X_{1: 2}^{\prime}, X_{1: 2}^{\prime} \leq{ }_{\mathrm{hr}} X_{1: 1}^{\prime}$. Because $\mathbf{p} \leq{ }_{\mathrm{hr}} \mathbf{q}$, by Theorem 3.4 we conclude that $X_{1: N_{1}}^{\prime} \leq \mathrm{hr} Y_{1: N_{2}}^{\prime}$.

\section{Acknowledgment}

The authors wish to thank three anonymous referees for their useful comments and suggestions which improved the presentation of the paper. 


\section{References}

[1] M. Asadi and I. Bayramoglu, "The mean residual life function of a $k$-out-of- $n$ structure at the system level," IEEE Transactions on Reliability, vol. 55, no. 2, pp. 314-318, 2006.

[2] J. Navarro, N. Balakrishnan, and F. J. Samaniego, "Mixture representations of residual lifetimes of used systems," Journal of Applied Probability, vol. 45, no. 4, pp. 1097-1112, 2008.

[3] I. Bairamov and B. C. Arnold, "On the residual lifelengths of the remaining components in an $n-k+1$ out of $n$ system," Statistics $\mathcal{E}$ Probability Letters, vol. 78, no. 8, pp. 945-952, 2008.

[4] M. Asadi and S. Goliforushani, "On the mean residual life function of coherent systems," IEEE Transactions on Reliability, vol. 57, no. 4, pp. 574-580, 2008.

[5] M. K. Sadegh, "A note on the mean residual life function of a coherent system with exchangeable or nonidentical components," Journal of Statistical Planning and Inference, vol. 141, no. 9, pp. 3267-3275, 2011.

[6] S. Eryilmaz, "On residual lifetime of coherent systems after the $r$ th failure," Statistical Papers. In press.

[7] P. J. Boland, "Signatures of indirect majority systems," Journal of Applied Probability, vol. 38, no. 2, pp. 597-603, 2001.

[8] F. J. Samaniego, System Signatures and Their Applications in Engineering Reliability, Springer, New York, NY, USA, 2007.

[9] S. Eryilmaz, "The number of failed components in a coherent system with exchangeable components," IEEE Transactions on Reliability, vol. 61, no. 1, Article ID 6030957, pp. 203-207, 2012.

[10] E. T. Salehi, M. Asadi, and S. Eryilmaz, "On the mean residual lifetime of consecutive $k$-out-of- $n$ systems," Test, pp. 1-23, 2011.

[11] S. Kochar, H. Mukerjee, and F. J. Samaniego, "The "signature" of a coherent system and its application to comparisons among systems," Naval Research Logistics, vol. 46, no. 5, pp. 507-523, 1999.

[12] M. Shaked and J. G. Shanthikumar, Stochastic Oders, Springer, New York, NY, USA, 2007.

[13] J. Navarro and R. Rubio, "Computations of signatures of coherent systems with five components," Communications in Statistics. Simulation and Computation, vol. 39, no. 1, pp. 68-84, 2010. 


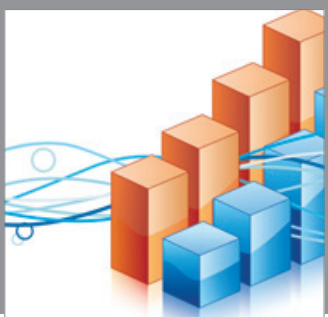

Advances in

Operations Research

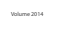

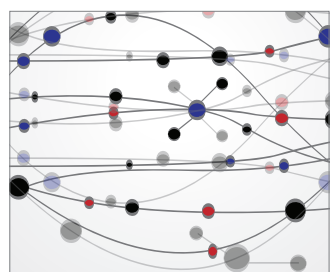

\section{The Scientific} World Journal
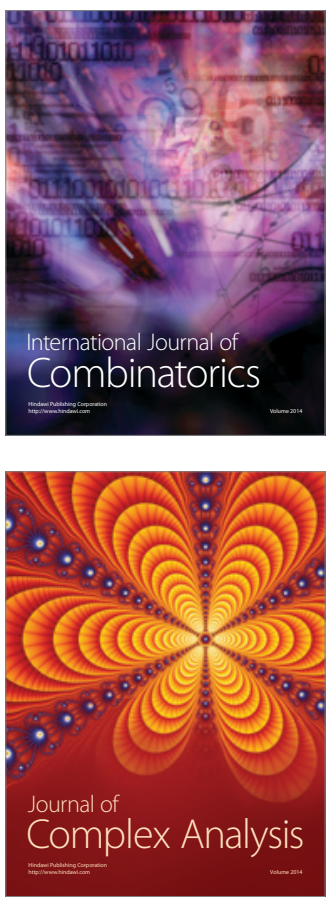

International Journal of

Mathematics and

Mathematical

Sciences
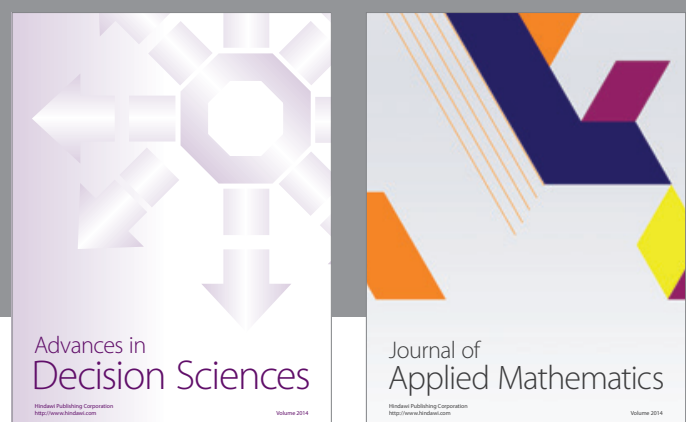

Journal of

Applied Mathematics
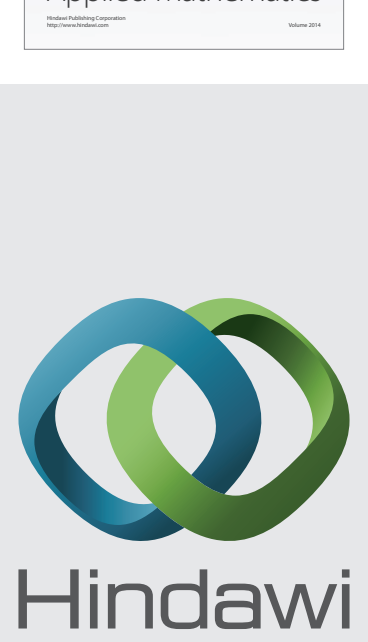

Submit your manuscripts at http://www.hindawi.com
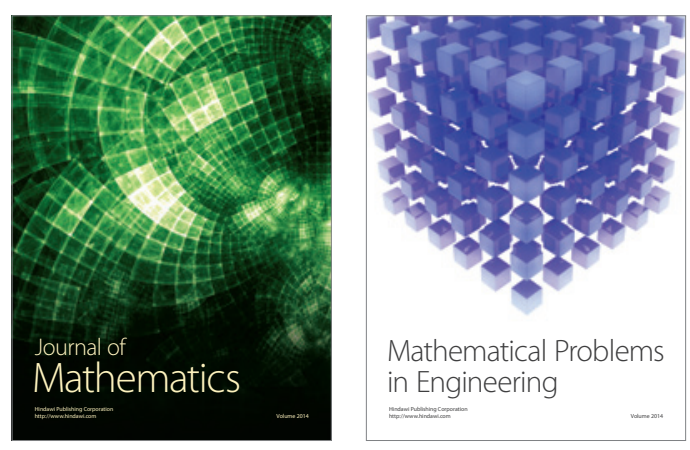

Mathematical Problems in Engineering
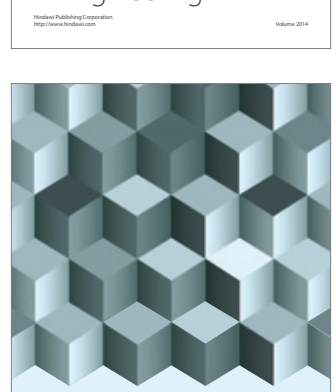

Journal of

Function Spaces
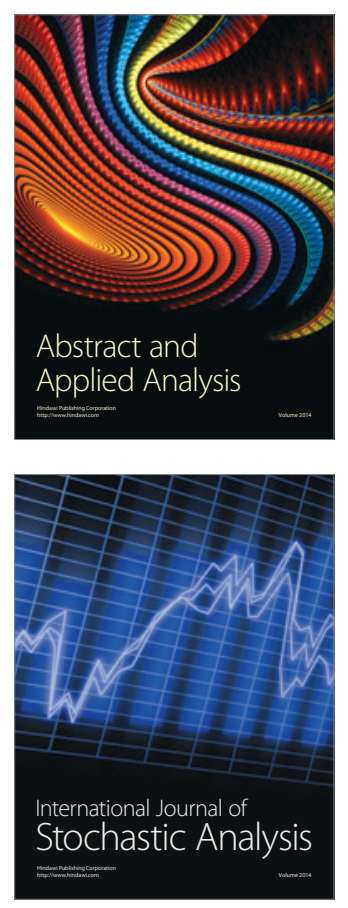

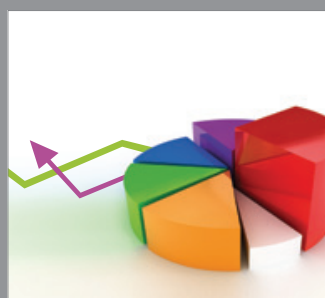

ournal of

Probability and Statistics

Promensencen
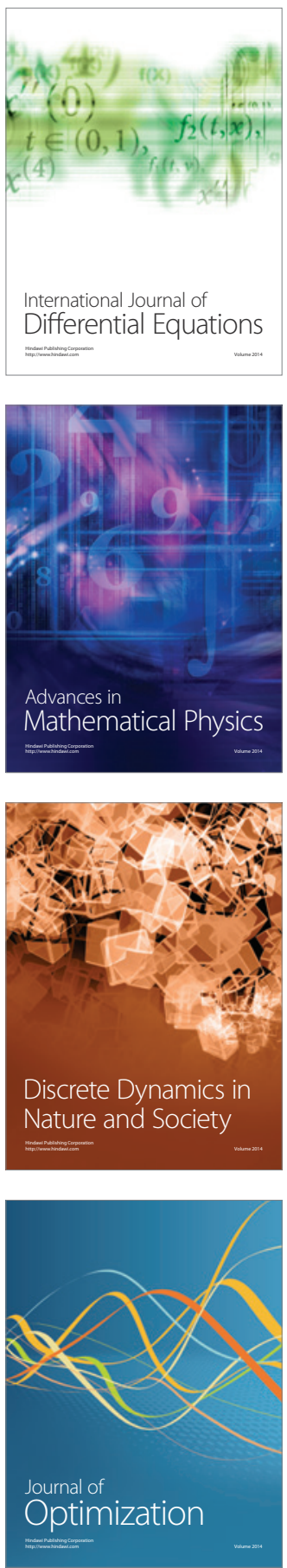